\title{
A SURJECTIVE CHARACTERIZATION OF DUGUNDJI SPACES
}

\author{
BURKHARD HOFFMANN ${ }^{1}$
}

\begin{abstract}
It is shown that the class of Dugundji spaces coincides with the class of continuous images of the generalized Cantor set by maps satisfying the zero-dimensional lifting property. It follows that each point in a Dugundji space has a neighbourhood base of Dugundji spaces.
\end{abstract}

Introduction. In [6] Pekczyński introduced the classes of Dugundji spaces and Milutin spaces. The fact that these classes coincide for compact spaces of weight not exceeding $\omega_{1}$ was established in the joint paper of Ditor and Haydon [2]. A major breakthrough in the characterization of Dugundji spaces and Milutin spaces was achieved by R. Haydon [4] showing that every Dugundji space is a Milutin space and that the notions of Dugundji space and absolute extensor for compact zero-dimensional spaces (AE(0-dim)) are equivalent. Recently in his paper [7] Shchepin gave another characterization of the class of Dugundji spaces and produced an example of a zero-dimensional space of weight $\omega_{2}$ that is a non-Dugundji Milutin space.

In this paper we shall consider continuous surjections satisfying the zero-dimensional lifting property (z.d.l.p.) which will lead to a new characterization of Dugundji spaces. At the same time our method yields topological information about surjective maps allowing a regular averaging operator (r.a.o.) and about the local structure of Dugundji spaces.

In his monograph Pekzyński asked for a topological characterization of continuous surjections of compact metrizable spaces that allow an r.a.o. [6, Problem 3, p. 65]. An answer was given by S. Z. Ditor [1, Theorem 3.4] in terms of existence of lower semicontinuous set-valued sections. Here our solution to Pekzyński's problem is of a neater, purely topological nature just involving continuous maps and compact spaces (see Corollary 1).

A locally Dugundji space is known to be a Dugundji space [6, Proposition 6.3] and Pelczyński raised the question whether the converse of this statement holds true [6, Problem 18, p. 70]. The corollary to our main result entails that the answer to this problem is in the affirmative.

Throughout this paper our terminology is standard; all definitions and

Received by the editors January 30, 1978 and, in revised form, October 12, 1978.

AMS (MOS) subject classifications (1970). Primary 54C40, 54C55; Secondary 54C10.

Key words and phrases. Zero-dimensional lifting property, regular averaging operator, Dugundji space.

${ }^{1}$ This research was supported in part by the German Academic Exchange Service under contract no. $425 / 009 / 318 / 5$. 
notations can be found in [4] or [6]. D will denote the two point discrete space $\{0,1\}$ and $I$ the closed unit interval with the usual topology.

Finally we record the following terminology. For a compact space $S$ we write $C(S)$ for the space of continuous real-valued functions on $S$ equipped with the topology induced by the supremum norm. The dual $M(S)$ is, as usual, identified with the set of signed, regular Borel measures on $S$ and $M(S)$ always carries the $\sigma(M(S), C(S))$-topology. For $s \in S$ the point measure at $s$ is denoted by $\delta_{s}$.

A continuous surjection $\psi: S \rightarrow T$ of compact spaces $S, T$ is said to allow a regular averaging operator or r.a.o., if there is a positive operator $u: C(S) \rightarrow$ $C(T)$ such that $u(f \circ \psi)=f$ for all $f \in C(T)$. Note that the operator $u$ is necessarily of norm 1. A Milutin space is defined as the continuous image of some generalized Cantor set $\mathrm{D}^{A}$ by a map allowing an r.a.o.

Zero-dimensional lifting property. Before introducing a new piece of terminology let us recall a selection theorem of $E$. Michael [5, Theorem 2] which we shall use as a basic tool.

LEMMA 1. If $R$ is a zero-dimensional paracompact space and $S$ is a complete metric space, then every lower semicontinuous function from $R$ into the closed, nonempty subsets of $S$ admits a continuous selection.

In this paper we are interested in lower semicontinuous functions which arise by assigning to a measure its support. This result appears as Proposition 3.1 in [1] which, for convenience of the reader, we record here again.

LEMMA 2. Let $S$ be a compact space. Then the mapping from $M(S)$ to the closed subsets of $S$ which takes a measure into its support is lower semicontinuous.

Finally the following notation will play a central role here.

Definition. Let $S, T$ be compact spaces and $\psi: S \rightarrow T$ a continuous surjection. Then $\psi$ is said to satisfy the zero-dimensional lifting property or $z . d . l . p$. if for any zero-dimensional compact space $R$ and for any continuous map $\phi: R \rightarrow T$ there exists a continuous map $\theta: R \rightarrow S$ such that the diagram commutes, i.e., $\psi \circ \theta=\phi$.

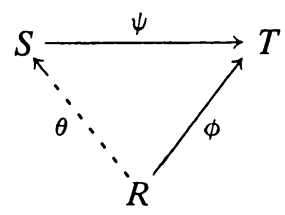

Let us start the investigation of z.d.l.p. by establishing some elementary observations.

We notice that the notion of z.d.l.p. is stable under taking finite compositions, and lifting a given map coordinatewise immediately yields that a 
product map $\left(\psi_{a}\right)_{a \in A}: \Pi\left\{S_{a}: a \in A\right\} \rightarrow \Pi\left\{T_{a}: a \in A\right\},\left(s_{a}\right)_{a \in A} \mapsto\left(\psi_{a}\left(s_{a}\right)\right)_{a \in A}$, satisfies the z.d.l.p., if each coordinate map $\psi_{a}: S_{a} \rightarrow T_{a}(a \in A)$ has this property.

Let us agree to call a property of a surjection $\psi: S \rightarrow T$ hereditary if for each closed subset $T_{0}$ of $T$, the induced restriction

$$
\psi \mid \psi^{-1}\left(T_{0}\right): \psi^{-1}\left(T_{0}\right) \rightarrow T_{0}
$$

has the same property.

Evidently, satisfying the z.d.l.p. is a hereditary property. Slightly less obvious is the fact that allowing an r.a.o. is a hereditary property too. To this end let us recall a characterization of r.a.o. which is a straightforward consequence of Proposition 4.1 of Pelczyński's monograph [6] and seems to be more suitable for our topological framework than the standard one given in the introduction.

A continuous surjection $\psi: S \rightarrow T$ of compact spaces $S, T$ admits an r.a.o. if and only if there exists a continuous map $\mu$ of $T$ into $M(S)$ taking each $t \in T$ into a probability measure $\mu_{t}$ such that the support of $\mu_{t}$ is contained in the fibre $\psi^{-1}(t)$.

Now suppose we are given a continuous surjection $\psi: S \rightarrow T$ allowing an r.a.o. and a closed subset $T_{0}$ of $T$. Then for all $t \in T_{0}$ the probability measure $\mu_{t}$ is supported by the inverse image $\psi^{-1}\left(T_{0}\right)$ and therefore can be canonically identified with a probability measure on $\psi^{-1}\left(T_{0}\right)$. Utilizing Pekzyński's result again yields that allowing an r.a.o. is a hereditary property.

An application of Lemmas 1 and 2 enables us to relate maps satisfying the z.d.l.p. with maps allowing an r.a.o.

Proposition 1. Let $S, T$ be compact metrizable spaces and $\psi: S \rightarrow T$ a continuous surjection allowing an r.a.o. $u: C(S) \rightarrow C(T)$. Then $\psi$ satisfies the z.d.l.p.

Proof. Since $u$ is an r.a.o. for $\psi$, the continuous map that assigns to each $t \in T$ the probability measure $\delta_{t} \circ u$ is such that the support of $\delta_{t} \circ u$ is contained in the fibre $\psi^{-1}(t)$ [6, Proposition 4.1]. Now let us assume we are given a zero-dimensional compact space $R$ and a continuous map $\phi: R \rightarrow T$. By Lemma 2 the set-valued function that takes each $r \in R$ into the support of the probability measure $\delta_{\phi(r)} \circ u$ satisfies the hypotheses of Lemma 1 and so it admits a continuous selection $\theta: R \rightarrow S$. In particular, $\theta(r)$ is an element of the fibre $\psi^{-1}(\phi(r))$ for all $r \in R$, i.e., $\phi=\psi \circ \theta$.

In order to infer that the restriction to metrizability in the preceding proposition is indeed essential we follow Shchepin's terminology and denote by $\exp _{n}(S)$ the space of all nonempty subsets of $S$ of cardinality $<n$ furnished with the finite topology (see [7] for more details). In passing we mention that the space $\exp _{2}\left(D^{\omega_{2}}\right)$ was exhibited in [7] as the first known example of a Milutin space that is not a Dugundji space. The fact that for any set $A$ the space $\exp _{2}\left(D^{A}\right)$ is a Milutin space is an easy consequence of the 
following observation. For a compact space $S$ the natural surjection $S \times S \rightarrow$ $\exp _{2}(S),(x, y) \rightarrow\{x, y\}$, is continuous and allows an r.a.o., which is given by the continuous map taking each element $\{x, y\}$ of $\exp _{2}(S)$ into the probability measure $\frac{1}{2}\left(\delta_{(x, y)}+\delta_{(y, x)}\right)$.

REMARK 1. The natural surjection $\psi: \mathbf{D}^{\omega_{1}} \times \mathbf{D}^{\omega_{1}} \rightarrow \exp _{2}\left(\mathbf{D}^{\omega_{1}}\right),(x, y) \rightarrow$ $\{x, y\}$, allows an r.a.o., but does not satisfy the z.d.l.p.

PrOof. By the above mentioned result it remains to verify the second part of the statement. Let us suppose the contrary. Since $\exp _{2}\left(D^{\omega_{1}}\right)$ is a zero-dimensional compact space, the z.d.l.p. of $\psi$ entails that $\psi$ is a retraction. Composing a continuous section for $\psi$ with the canonical projection $\mathbf{D}^{\omega_{1}} \times$ $\mathbf{D}^{\omega_{1}} \rightarrow \mathbf{D}^{\omega_{1}}$ onto the first coordinate yields a continuous selection $\sigma: \exp _{2}\left(\mathbf{D}^{\omega_{1}}\right)$ $\rightarrow \mathbf{D}^{\omega_{1}}$, i.e., $\sigma(\{x, y\}) \in\{x, y\}$ for all elements $\{x, y\}$ of $\exp _{2}\left(\mathbf{D}^{\omega_{1}}\right)$. By a theorem of G. S. Young [8, Theorem 3] the existence of such a continuous selection implies that $\mathbf{D}^{\omega_{1}}$ admits a linear order such that the order topology coincides with the original topology. But this is impossible, since a linearly ordered dyadic space is known to be homeomorphic to a subset of the real line (see [3, Corollary 7]) and thus, in particular, it is metrizable.

Although the proof of Young's theorem cited above is not included in her essay, we refrain from giving the rather lengthy verification showing explicitly that the existence of a continuous selection $\sigma$ : $\exp _{2}\left(D^{\omega_{1}}\right) \rightarrow D^{\omega_{1}}$ induces a contradiction. Moreover, the reader who is familiar with the techniques developed by Shchepin can find an implicit mention of this result on p. 164 of [7].

Let us now proceed with the investigation of the interrelationship of z.d.l.p. and r.a.o. A converse of Proposition 1 can be proved without any restriction to metrizability.

Proposition 2. Let $S, T$ be compact spaces and $\psi: S \rightarrow T$ a continuous surjection satisfying the z.d.l.p. Then $\psi$ allows an r.a.o.

Proof. We may assume $T$ to be a subset of some $\mathbf{I}^{A}$. Since $\mathbf{I}^{A}$ is a Milutin space [6, Theorem 5.6] there exists some continuous surjection $\phi: D^{B} \rightarrow I^{A}$ allowing an r.a.o. Let $R$ be the compact, zero-dimensional space $\phi^{-1}(T)$ and, for simplicity, write $\phi: R \rightarrow T$ for the restricted map as well. Since allowing an r.a.o. is a hereditary property we deduce that $\phi: R \rightarrow T$ admits an r.a.o. $u$, say. By hypothesis there exists a continuous lifting $\theta: R \rightarrow S$ such that $\psi \circ \theta=\phi$. A simple calculation shows that the operator $v: C(S) \rightarrow C(T)$, defined by $v(f):=u(f \circ \theta)$ for all $f \in C(S)$, is indeed an r.a.o. for $\psi$.

Combining Propositions 1 and 2 yields the following equivalence.

COROllaRY 1. Let $\psi: S \rightarrow T$ be a continuous surjection of compact metrizable spaces. Then $\psi$ allows an r.a.o. if and only if $\psi$ satisfies the z.d.l.p.

The main result. As in the definition of a Milutin space we restrict our attention to continuous surjections satisfying the z.d.l.p. and having as 
domain space some $\mathrm{D}^{4}$. Obviously, Proposition 2 shows that the class of spaces which occur as images of such maps is contained in the class of Milutin spaces. For further investigation it seems convenient to introduce the following notation.

We agree to call a compact space $S$ a Michael space if there exists a continuous surjection from some $\mathrm{D}^{4}$ onto $S$ satisfying the z.d.l.p.

Proposition 1 and the fact that a compact metrizable space is a Milutin space (see [6, Theorem 5.6]) entail that the class of Michael spaces contains all compact metrizable spaces. The observations following the definition of the z.d.l.p. show that the class of Michael spaces is closed under taking arbitrary products and images by continuous maps satisfying the z.d.l.p. We have just proved the following.

LEMMA 3. Any image of an arbitrary product of compact metrizable spaces by a continuous map satisfying the z.d.l.p. is a Michael space.

THEOREM. The class of Michael spaces coincides with the class of Dugundji spaces.

Proof. By Haydon's result [4] it is enough to show the equivalence of the terms "AE(0-dim)" and "Michael space".

So let us assume that $X$ is a Michael space and let $\psi: D^{A} \rightarrow X$ be a continuous surjection satisfying the z.d.l.p. Suppose we are given a compact, zero-dimensional space $T$, a closed subspace $S$ of $T$ and a continuous map $\phi$ : $S \rightarrow X$. By hypothesis there exists a continuous lifting $\theta: S \rightarrow D^{A}$ such that $\psi \circ \theta=\phi$. Certainly, D is an $\mathrm{AE}(0-\mathrm{dim})$ and continuously extending maps coordinatewise immediately yields that $\mathrm{D}^{4}$ is an $\mathrm{AE}(0-\mathrm{dim})$ too. (Alternatively, recall that a product of compact metrizable spaces is a Dugundji space [6, Proposition 6.5] or, equivalently, by Haydon's characterization [4] an $\mathrm{AE}\left(0\right.$-dim).) Hence there is a continuous extension $\bar{\theta}: T \rightarrow \mathrm{D}^{A}$ and the restriction of $\bar{\theta}$ to $S$ coincides with $\theta$. Consequently, $\psi \circ \bar{\theta}$ is a continuous extension of $\phi$.

Conversely, let $X$ be an $\mathrm{AE}(0-\mathrm{dim})$ which we may assume to be a subspace of some $\mathrm{I}^{A}$. By Lemma 3 there exists some continuous surjection $\psi: \mathrm{D}^{B} \rightarrow \mathrm{I}^{A}$ satisfying the z.d.l.p. Let $S$ be the closed subspace $\psi^{-1}(X)$ of $\mathbf{D}^{B}$ and $\phi$ : $S \rightarrow X$ the restriction of $\psi$ to $S$. Since satisfying the z.d.l.p. is a hereditary property, the restriction $\phi$ of $\psi$ satisfies the z.d.l.p. too. By hypothesis $\phi$ allows a continuous extension $\bar{\phi}: \mathbf{D}^{B} \rightarrow X$ and, obviously, $\bar{\phi}$ preserves the z.d.l.p. This shows that $X$ is a Michael space.

REMARK 2. Following up the observations of Remark 1 it seems interesting to notice that although the natural surjection $\psi: \mathbf{D}^{\omega_{1}} \times \mathbf{D}^{\omega_{1}} \rightarrow \exp _{2}\left(D^{\omega_{1}}\right)$ does not satisfy the z.d.l.p. there exists a continuous surjection $D^{\omega_{1}} \rightarrow \exp _{2}\left(D^{\omega_{1}}\right)$ satisfying the z.d.l.p. For the map $\psi$ allows an r.a.o. and so $\exp _{2}\left(D^{\omega_{1}}\right)$ is a Milutin space of weight $\omega_{1}$ and hence by the result of Ditor and Haydon [2] 
quoted in the introduction a Dugundji space or, equivalently, by our theorem a Michael space.

Our surjective characterization of Dugundji spaces yields as a corollary a positive answer to Problem 18 of Pekczyński’s book [6].

Corollary 2. If $S$ is a Dugundji space and $T$ a closed $G_{\delta}$-set in $S$, then $T$ is a Dugundji space. In particular, each point of $S$ has a neighbourhood base of Dugundji spaces.

Proof. If $S$ is metrizable the statement is trivial. So let us assume there exists an uncountable set $A$ and a continuous surjection $\psi: D^{A} \rightarrow S$ satisfying the z.d.l.p. The inverse image $\psi^{-1}(T)$ is a closed $G_{\delta}$-set in $\mathbf{D}^{A}$ which is known to be homeomorphic to $\mathrm{D}^{A}\left[3\right.$, Theorem 6]. The restriction of $\psi$ to $\psi^{-1}(T)$ maps continuously onto $T$ and inherits the z.d.l.p. from $\psi$.

ACKNOWLEDGEMENT. I should like to thank Dr. R. G. Haydon for suggesting this piece of research to me and the referee for bringing Ditor's paper [1] to my attention.

\section{REFERENCES}

1. S. Z. Ditor, Averaging operators in $C(S)$ and lower semicontinuous sections of continuous maps, Trans. Amer. Math. Soc. 175 (1973), 195-208.

2. S. Ditor and R. Haydon, On absolute retracts, $P(S)$, and complemented subspaces of $C\left(D^{\omega_{1}}\right)$, Studia Math. 56 (1976), 243-251.

3. B. A. Efimov, Dyadic bicompacta, Trans. Moscow Math. Soc. 14 (1965), 229-267.

4. R. Haydon, On a problem of Pelczyniski: Milutin spaces, Dugundji spaces and AE(0-dim), Studia Math. 52 (1974), 23-31.

5. E. Michael, Selected selection theorems, Amer. Math. Monthly 63 (1956), 233-238.

6. A. Pelczyński, Linear extensions, linear averagings, and their applications to linear topological classification of spaces of continuous functions, Dissertationes Math. (Rozprawy Mat.) 58 (1968).

7. E. V. Shchepin, Topology of limit spaces of uncountable inverse spectra, Russian Math. Surveys 315 (1976), 155-191.

8. G. S. Young, Continuous selections on locally compact separable metric spaces, set-valued mappings, selections and topological properties of $2^{X}$, Lecture Notes in Math., vol. 171, SpringerVerlag, Berlin and New York, 1970, pp. 102-110.

MATHEMATICAL INSTITUTE, UNIVERSITY OF OXFORD, OXFORD, ENGLAND 